\title{
Characterizing ultralow emittance electron beams using structured light fields
}

\author{
Andreas Seidel@ ${ }^{1,2,{ }^{*}}$ Jens Osterhoff, ${ }^{3}$ and Matt Zepf ${ }^{1,2}$ \\ ${ }^{1}$ Friedrich-Schiller-Universität, Fürstengraben 1, 07743 Jena, Germany \\ ${ }^{2}$ Helmholtz-Institut Jena, Fröbelstieg 3, 07743 Jena, Germany \\ ${ }^{3}$ Deutsches Elektronen-Synchrotron DESY, Notkestraße 85, 22607 Hamburg, Germany
}

(Received 2 July 2020; accepted 6 January 2021; published 20 January 2021)

\begin{abstract}
Novel schemes for generating ultralow emittance electron beams have been developed in past years and promise compact particle sources with excellent beam quality suitable for future high-energy physics experiments and free-electron lasers. Current methods for the characterization of low emittance electron beams such as pepperpot measurements or beam focus scanning are limited in their capability to resolve emittances in the sub $0.1 \mathrm{~mm}$ mrad regime. Here we propose a novel, highly sensitive method for the single shot characterization of the beam waist and emittance using interfering laser beams. In this scheme, two laser pulses are focused under an angle creating a gratinglike interference pattern. When the electron beam interacts with the structured laser field, the phase space of the electron beam becomes modulated by the laser ponderomotive force and results in a modulated beam profile after further electron beam phase advance, which allows for the characterization of ultralow emittance beams. 2D PIC simulations show the effectiveness of the technique for normalized emittances in the range of $\epsilon_{n}=[0.01,1] \mathrm{mm} \mathrm{mrad}$.
\end{abstract}

DOI: $10.1103 /$ PhysRevAccelBeams.24.012803

\section{INTRODUCTION}

The emittance of an electron beam is one of its most important quality measures for many applications. The emittance describes the volume occupied by the electron beam in six-dimensional phase space [1]. The transverse emittance of the beam determines the smallest spot size that can be achieved for a given focusing arrangement and hence influences the luminosity in High Energy Physics experiments. Another area of importance is in determining the gain of free-electron lasers through the Pierce parameter [2].

In linear accelerators the injection of low emittance electron bunches into the accelerating structure is of prime importance. Significant improvements in radio-frequency (rf) guns with laser photocathodes allow a small initial source size and limit the emittance growth in the early stages using strong accelerating fields of several tens of $\mathrm{MV} / \mathrm{m}$. Using this approach $\mathrm{GeV}$ level linear accelerators (linacs) currently achieve a normalized transverse emittance $\epsilon_{n}=\beta_{e} \gamma_{e} \epsilon\left(\beta_{e}=v_{e} / c, \gamma_{e}\right.$-electron gamma factor, and $\epsilon$-geometric beam emittance) of $0.002 \mathrm{~mm}$ mrad [3]

*seidel.andreas@uni-jena.de

Published by the American Physical Society under the terms of the Creative Commons Attribution 4.0 International license. Further distribution of this work must maintain attribution to the author(s) and the published article's title, journal citation, and DOI. for a bunch with less than ten electrons, $0.10 \mathrm{~mm}$ mrad [4] for a bunch with a charge of $2 \mathrm{pC}$ and $0.89 \mathrm{~mm}$ mrad [5] for a bunch with a charge of $1 \mathrm{nC}$, with continuous strides being made to achieve even lower emittances.

Laser plasma wakefield accelerators (LWFA) [6,7] feature very large accelerating fields reaching $100 \mathrm{GV} / \mathrm{m}$ or more. In principle these large fields result in the electron beam becoming highly relativistic over a propagation length of a fraction of a $\mathrm{mm}$ and consequently provide a promising route to achieving extremely low emittances. As in the case of rf accelerators, the injection volume is a key determinant of the final emittance. This is especially true for low charge beams, where emittance growth due to space charge is negligible. To date most laser plasma wakefield experiments with a dedicated injection procedure have used either ionization [8,9], downramp [10] or colliding pulse injection [11] and transverse emittances of similar magnitude to $\mathrm{rf}$ linear accelerators have been attained $\left(\epsilon_{n}<0.2 \mathrm{~mm} \operatorname{mrad}[12,13]\right)$.

Further improvements can be realized if the phase space injection volume and field perturbations are minimized. A promising approach here is the plasma photocathode, which combines the well-defined volume achievable by ionization injection with a separate injection laser with a particle-beam-driven wakefield [14]. This approach allows the laser intensity used for ionization injection to be minimized resulting in a low initial momentum spread. Additionally, emittance growth is reduced by eliminating the powerful laser driving the wakefield structure and 
thus the unavoidable field fluctuations introduced by an oscillating driver field. Simulations have predicted that an emittance of the order of $10 \mu \mathrm{m} \mathrm{mrad}$ is feasible [15].

The potential performance gains by such a marked reduction in emittance are highly desirable and experiments are under way to explore the development of lower emittance beams. Current measurement techniques which are being used for monochromatic and polychromatic electron beams such as beam focus scanning have shown to be capable of measuring emittances down to $\epsilon_{n} \approx$ $0.2 \mathrm{mmmrad}[12,13]$. A further improvement of the detection limit using beam focus scanning techniques is conceivable. However, their practical realization is quickly becoming challenging for smaller emittances and large setups are required. A novel knife-edge based measurement characterization is capable of measuring electron beam emittances down to $0.002 \mathrm{~mm}$ mrad [3], but was limited to monoenergetic and low energy $(<1 \mathrm{MeV})$ electron beams. Hence new approaches are needed to characterize the next generation of electron beams.

In the following we describe a method that spatially modulates the transverse momentum distribution of the electron beam on $\mu \mathrm{m}$ spatial scale using a laser beam and allows the size of the electron beam waist to be determined from the observed modulation on a simple beam monitor screen downstream. The sensitivity can be adjusted via the spatial frequency of the laser interference pattern, allowing e-beam waists from tens of $\mathrm{nm}$ to $\mu \mathrm{m}$ scale to be determined. The emittance range that can be covered by this technique is also determined by the divergence of the ebeam and is compatible with a large emittance parameter range from the $\mathrm{mm}$ mrad of current linacs to the $\mu \mathrm{m} \mathrm{mrad}$ anticipated for novel injection schemes. Measurements of small spatial scales with laser interference structures is a viable approach to assess the quality of electron beams and has been demonstrated in beam-size monitors, where in contrast to wire scanners the fine wire is replaced by a "laser-wire" formed by interfering lasers in the so-called Shintake monitor [16]. It can resolve nm-spatial scale electron beam foci using a scanning technique. While this method requires a measurement of the electron bunches at focus, which can be an immense effort for polychromatic electron beams, our proposal is compatible with broadband diverging beams and also allows a determination of the slice emittance.

\section{THEORETICAL MODEL}

The proposed measurement scheme is shown schematically in Fig. 1. Two laser beams with wavelength $\lambda$ cross under an angle $2 \cdot(90-\theta)$ to form an interference pattern with a periodicity $d=\lambda /(2 \cos \theta)$ at a distance $z$ from the (a)

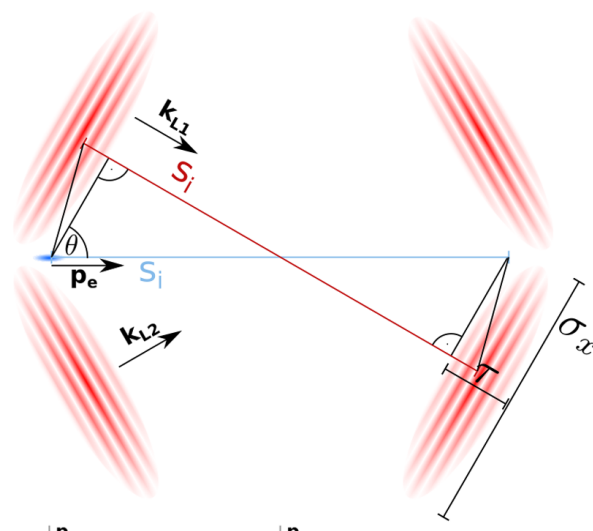

(b)

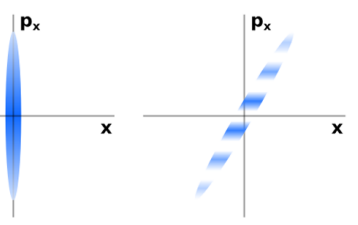

(c)

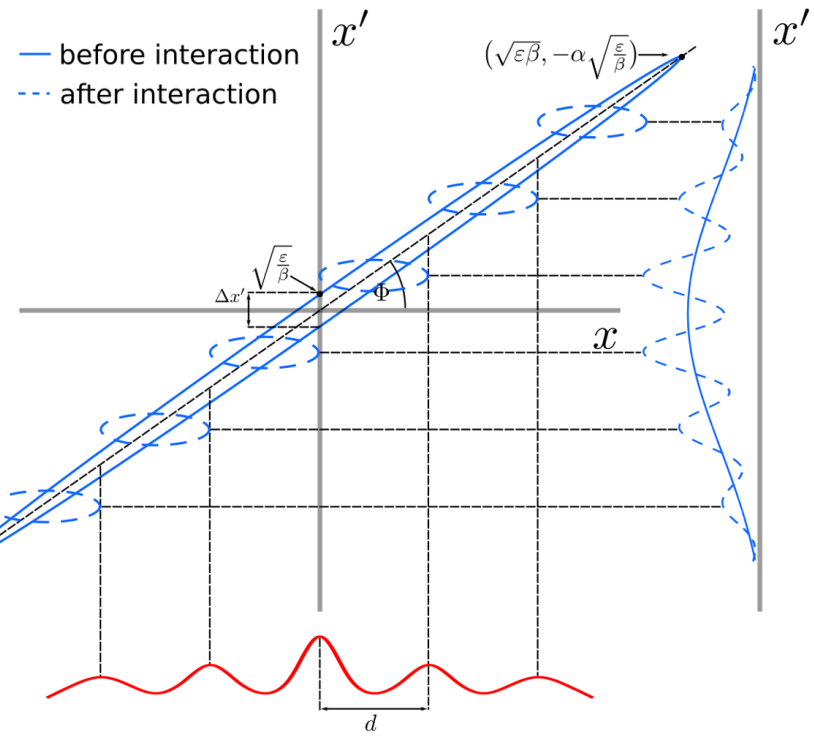

FIG. 1. (a) Schematic of the measurement setup showing two laser beams crossing under an angle $2 \cdot(90-\theta)$ to form an interference pattern in the interaction region (Fig. 2). After the laser-electron interaction the imparted momentum modulation evolves into a transverse density modulation that can be observed on a downstream beam monitor screen and allows the e-beam waist size to be determined. Inclusion of a magnet behind interaction allows the emittance of monochromatic beam slices to be measured even for beams with a large projected energy spread. (b) Schematic of the beam momentum distribution at the waist, the interaction point and after free space propagation (from left to right). (c) Transverse electron phase space before (solid) and after laser-electron interaction (dashed). The red line indicates the intensity profile of the two interfering laser beams at the corresponding transverse position $\mathrm{x}$ at the interaction point. The ponderomotive force shifts the transverse momentum of the electrons. For an optimum laser intensity the momentum transfer is matched to the gradient of the phase space and shifts particles from regions of high laser intensity to regions of low intensity highlighted by the dashed ellipses. 


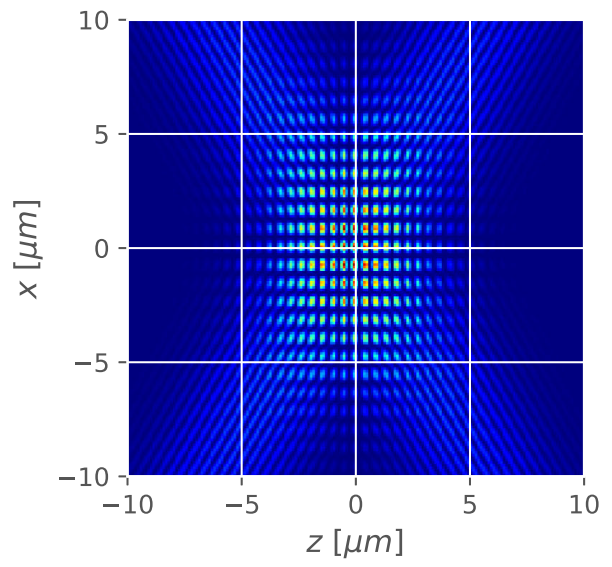

FIG. 2. Intensity pattern of the interference of the colliding laser pulses.

electron beam waist (Fig. 2). In the absence of the laser beam the unperturbed electron beam profile is visible on a measurement screen at a convenient location downstream of the interaction region. When the laser is switched on, the transverse momenta of the electrons are modulated by the field of the laser with the periodicity of the interference pattern allowing a modulated electron beam pattern to be observed on the screen.

The working principle is shown in greater detail in Fig. 1(c). The electron beam transverse dimension $x$ and divergence angle $x^{\prime}$ can be described as an ellipse [17] parametrized by TWISS parameters $\alpha, \beta, \gamma$ so that $\epsilon=\gamma x^{2}+2 \alpha x x^{\prime}+\beta x^{\prime 2}$. In this description the beam width $\sigma_{x}$ and angular spread $\sigma_{x^{\prime}}$ are given by $\sigma_{x}(z)=\sqrt{\beta(z) \epsilon}$ and $\sigma_{x^{\prime}}(z)=\sqrt{\gamma(z) \epsilon}$ as a function of the distance $z$ from the beam waist respectively and $\tan \Phi=d x^{\prime} / d x \propto 1 / z$. Note that while the ellipse has an overall width of $\sigma_{x^{\prime}}$ the slice width at a given transverse position $x$ can be significantly less $\Delta x^{\prime}=2 \sqrt{\frac{\epsilon}{\beta(z)}}$ and decreases with the propagation distance $\mathrm{z}$ from the waist. This implies that a visible modulation of the electron beam can be achieved by imparting a transverse kick to the electrons of $\sim \Delta x^{\prime}$, in which case the electron beam will exhibit areas of increased phase space density at certain values $x^{\prime}$ and reduced at others. As can be seen from Fig. 1(c), the separation of adjacent intensity peaks $d$ must be large enough to prevent the two adjacent regions with slice width $\Delta x^{\prime}=2 \sqrt{\epsilon / \beta(z)}$ from overlapping in $x^{\prime}$ space. At large values of $\alpha$ (i.e., far from the waist) the lower limit for the laser interference pattern period $d$ is therefore

$$
d>2 \frac{\sqrt{\epsilon \beta(z)}}{\alpha(z)} \approx 2 \frac{\epsilon}{\sigma_{x^{\prime}}}=2 \frac{\epsilon}{\sigma_{x^{\prime}}}=2 \sigma_{x}(0)=\text { const. }
$$

The condition for the minimum periodicity is therefore connected to the beam waist size and this condition is independent of the distance $\mathrm{z}$ from the electron beam waist where the measurement is taken. As we shall see in the following, the waist size can be determined from the modulation depth without the need for a direct measurement at the location of the beam waist itself. Combined with a simple measurement of the unperturbed beam divergence we can therefore use this method to accurately determine the emittance of the electron beam.

The laser imparts momentum to the electrons via the ponderomotive force $F_{p} \propto \nabla E^{2}$ of the laser which tends to push electrons from regions of high intensity to regions of low intensity. As can be seen from Fig. 1(c) the optimal modulation depth is achieved when electrons are shifted in transverse momentum by an amount that matches the gradient of the ellipse in phase space. In momentum terms this requirement can be expressed by

$$
\frac{\Delta p_{x}}{\Delta x}=\frac{d p_{x}}{d x}=p_{z} \tan \Phi \propto \frac{p_{z}}{z} .
$$

Assuming that the ponderomotive force is the dominant force in changing the particle momentum, we can derive the requirement

$$
\frac{d p_{x}}{d t d x} \stackrel{!}{=} \frac{F_{\mathrm{pond}}}{d x} .
$$

For a given gradient of the transverse momentum across the electron beam, an optimal laser intensity leads to the desired modulation of transverse momentum and, hence, maximum modulation visibility. The change in transverse momentum can be expressed in terms of the interaction time $t_{\text {int }}$ and laser intensity $I$ as

$$
\frac{d p_{x}}{d x}=\frac{\left\langle F_{\mathrm{pond}}\right\rangle t_{\mathrm{int}}\left(\gamma_{e}\right)}{d x} \propto \frac{d\langle I\rangle}{\omega^{2} d x} \frac{t_{\mathrm{int}}\left(\gamma_{e}\right)}{d x},
$$

where \langle\rangle is a time average over the duration of the interaction and $\omega$ the laser frequency. Since the intensity of the interference pattern drops from its maximum to zero over a distance of $d / 2$ and using Eq. (3) we obtain

$$
\frac{\Delta p_{x}}{\Delta x} \propto\langle I\rangle_{0} \cdot t_{\text {int }}\left(\gamma_{e}\right),
$$

where $\langle I\rangle_{0}$ is the time averaged intensity required to achieve the optimum beam modulation. Inserting into Eq. (2) we find the average interaction intensity to achieve the optimum modulation depth with

$$
\langle I\rangle_{0} \propto \frac{p_{z}}{z \cdot t_{\text {int }}\left(\gamma_{e}\right)} .
$$

As one would expect, the laser intensity increases for higher energy electron beams and decreases with increasing distance from the beam waist. The latter scaling can be 
simply understood, as the increased beam size with increasing $z$ reduces the gradient of transverse momentum. This corresponds to a shallower gradient of the emittance ellipse and a reduced momentum modulation required to be imparted by the laser. Note that the required laser energy also depends on the interaction length (ignoring beam size effects) $s_{i}=c \tau /(1-\sin (\theta))$, favoring shallow crossing angles if a sufficiently small $d$ is maintained. The required intensity can thus be kept subrelativistic, which diminishes the demands on the laser system.

Assuming an electron bunch with Gaussian transverse beam waist $\sigma_{x 0}$ and corresponding momentum $\sigma_{p x 0}$ we obtain

$$
n\left(x, p_{x}\right)=n_{e 0} \cdot \exp \left(-\frac{p_{x}^{2}}{2 \sigma_{p x 0}^{2}}-\frac{x^{2}}{2 \sigma_{x 0}^{2}}\right)
$$

for the density distribution at waist position. With Eqs. (2) and (3) we obtain

$$
\begin{aligned}
n\left(p_{x}\right)= & n_{e 0} \int_{-\infty}^{\infty} \exp \left(-\frac{\left(p_{x}\right)^{2}}{2 \sigma_{p x 0}^{2}}\right. \\
& \left.-\frac{\left(\left(p_{x}-\Delta p_{x}\right) /\left(p_{z} \tan \Phi\right)-x\right)^{2}}{2 \sigma_{x 0}^{2}}\right) d x,
\end{aligned}
$$

for the momentum space after the laser-electron interaction, where $\Delta p_{x}=\left\langle F_{\text {pond }}\right\rangle \cdot t_{\text {int }}\left(\gamma_{e}\right)$. The first term in the exponent represents the transverse divergence of the electron bunch, which is not affected by the interaction with the laser interference pattern. The second term describes the changes in the transverse momentum space caused by the ponderomotive force and indicates an increase in the peak height of the created modulations with decreasing values of $\sigma_{x 0}$.

\section{SIMULATION RESULTS}

2D simulations to test the scheme were conducted using the code EPOCH [18]. The simulations were performed in a moving box with the size of $z=320 \mu \mathrm{m}$ and $x=80 \mu \mathrm{m}$ with 20 cells/micron resolution in every dimension. The electron bunch, sampled by $8 \times 10^{5}$ macroparticles, had a longitudinal size of $\sigma_{z}=2 \mu \mathrm{m}$ and variable transverse size $\sigma_{x}$ and propagated along the $\mathrm{z}$ axis. The electron bunch charge was chosen at $150 \mathrm{fC}$ to ensure an interaction between laser and electron bunch without further charge related effects. The interaction laser was modeled as two pulses propagating linearly polarized in the y direction and with a duration $\tau_{\mathrm{FWHM}}=17 \mathrm{fs}$, spot size at interaction $\sigma_{\mathrm{FWHM}}=30 \mu \mathrm{m}$ at a wavelength of $\lambda=0.8 \mu \mathrm{m}$. The laser intensity distribution produced by the two interfering pulses is shown in Fig. 2 with the lasers propagating from the upper left side and the lower left side. The angle between the $\mathrm{z}$ axis and lasers is $90-\theta=30^{\circ}$.
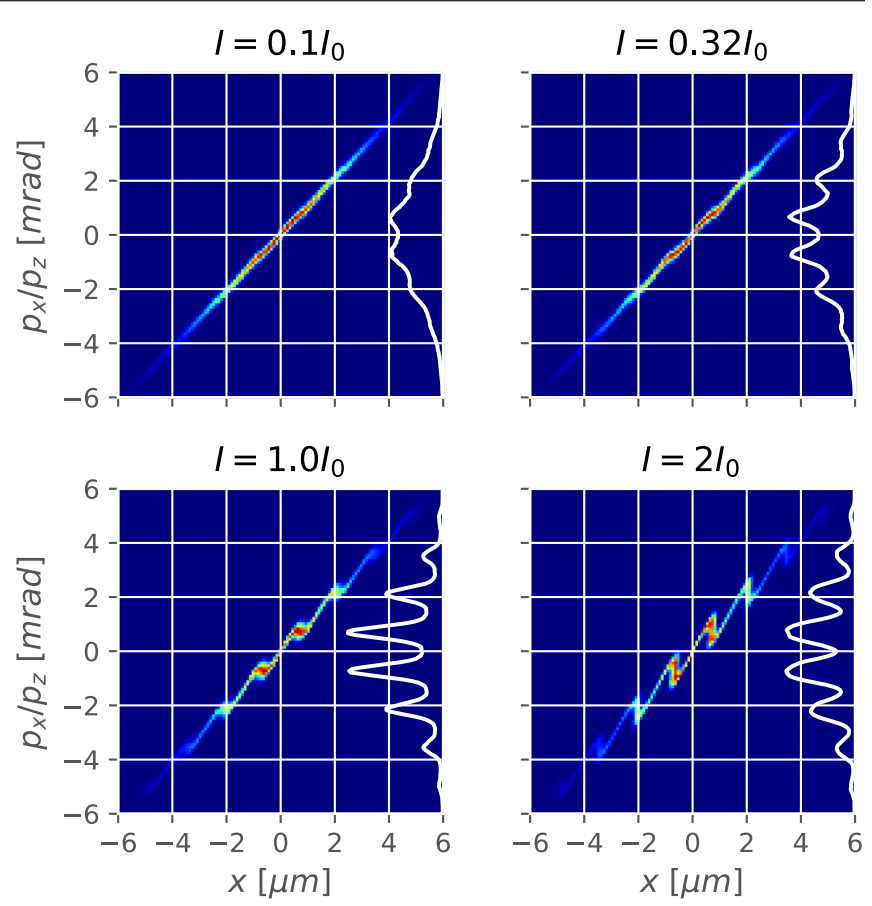

FIG. 3. Momentum space of electron beam $\left(\gamma_{e}=100\right.$, $\left.\sigma_{x}=100 \mathrm{~nm}, \sigma_{x^{\prime}}=2 \mathrm{mrad}, \sigma_{z}=2 \mu \mathrm{m}\right)$ after interaction with different laser intensities. As can be seen the modulation optimizes for a specific intensity and reduces again for higher values. White curve: transverse momentum space integrated along $\mathrm{x}$ (measured signal on detector).

Figure 3 shows the electron beam modulations for different laser intensities. The initial electron bunch parameters are $\gamma_{e}=100, \sigma_{x}=100 \mathrm{~nm}, \sigma_{x^{\prime}}=2 \mathrm{mrad}, \sigma_{z}=$ $2 \mu \mathrm{m}$ and $I_{0}=3.5 \times 10^{16} \mathrm{~W} / \mathrm{cm}^{2}$. The ponderomotive force imparts transverse momentum to the electrons and concentrates the electrons at certain propagation angles corresponding to the nodes of the interference pattern, resulting in an intensity modulation on the diagnostic. Although these laser intensities do not cause instantaneous changes in the position of the electrons, the electrons drift due to their transverse momentum during interaction in the interference grating. This finally leads to a blurring of the modulation signal on the detector. Our simulations have shown for an electron drift during the interaction of less than $10 \%$ of the laser interference pattern period $\mathrm{d}$, there is no measurable difference in the modulation.

The modulation is strongest for the matched intensity $I_{0}$ and decreases above and below this. For intensities that are too low, the perturbation is much smaller than the local slice angular spread $\Delta x^{\prime}$ (the ellipse width in the $x^{\prime}$ direction) leading to a negligible effect. At large intensities the electrons are displaced by much more than the local ellipse width and the effect is "smeared out." For a given set of experimental parameters, the peak laser intensity at which the optimal modulation depth occurs is shown in Fig. 4. With $P_{\text {Laser }} \approx I_{0} / 2 \cdot \pi \sigma^{2}$ the required laser power for this particular electron bunch and interaction point $1600 \mu \mathrm{m}$ 


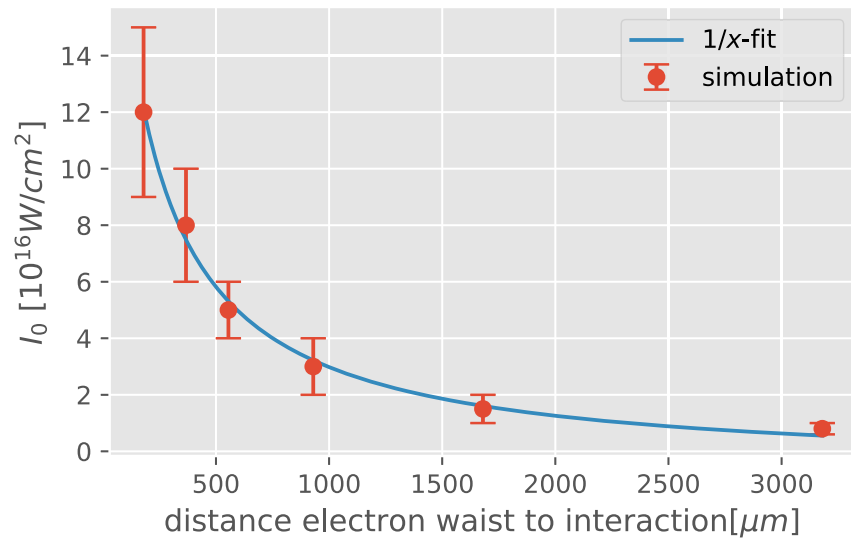

FIG. 4. Optimum peak intensity of a single laser used, to get strongest modulation signal in momentum space of the electron beam $\left(\gamma_{e}=100, \sigma_{x}=150 \mathrm{~nm}, \sigma_{x^{\prime}}=2 \operatorname{mrad}, \sigma_{z}=2 \mu \mathrm{m}\right)$ for various distances between electron beam being at waist and laserelectron interaction. The vertical bars indicate the intensity range over which the change in maximum modulation depth is less than $5 \%$.

behind the electron waist matched to a laser spot size $\sigma_{\mathrm{FWHM}}=30 \mu \mathrm{m}$ is $300 \mathrm{GW}$.

One might assume that this method places increasingly onerous requirements on the laser to make a measurement for beams with very high electron beam energy. Since the width of the ellipse and therefore the optimal intensity depends on the distance $\mathrm{z}$ from the beam waist as $1 / z$, allowing the optimal intensity to be controlled by the interaction geometry. Note that for limited available laser power there is no intrinsic requirement for the laser spot to be larger than the electron beam. In principle smaller spots can be used with scanning measurements to determine the emittance of the beam, therefore enabling measurements with lasers that are easily colocated with an electron beam. Note that any relative pointing jitter between laser interference pattern and electron bunch leads to the same results as the proposed scanning measurement. However, it must be ensured that the laser interference pattern remains in place until the whole electron bunch in the longitudinal direction has interacted with it.

As is clear from the discussion in the previous section and can be seen in Fig. 5 higher energy electron beams require a higher laser intensity for optimal modulation for otherwise identical parameters. The increase is quadratic in the Lorentz-factor $\gamma_{e}$ due to the relativistic contraction of the interaction length and the higher transverse momentum required to achieve the same ratio of $p_{x} / p_{z}$ on the beam electrons. It is important to note that at the optimum laser intensity the maximum modulation depth is independent of the electron bunch $\gamma_{e}$.

From the discussion above we can now formulate a measurement approach for calculating the desired e-beam characteristics. As is clear from Fig. 6 the change in the beam intensity modulation depends only on source size for

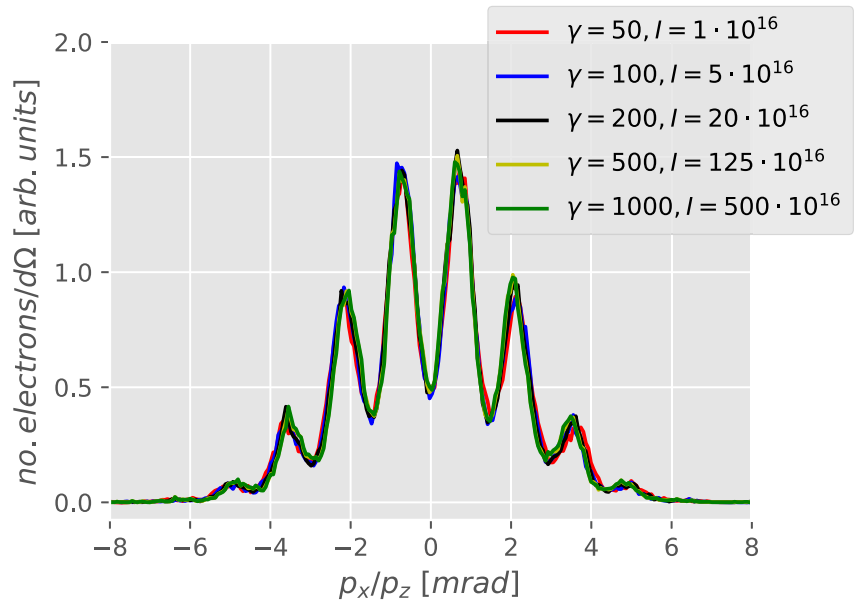

FIG. 5. Transverse momentum space of electron beams with different $\gamma_{e}\left(\sigma_{x^{\prime}}=2 \mathrm{mrad}, \sigma_{z}=2 \mu \mathrm{m}, \sigma_{x}=150 \mathrm{~nm}\right)$ after interaction. Laser intensity is optimized for maximum modulation depth.

fixed laser intensity with the beam modulation visible as long as the criterion from Eq. (1) is met. The proposed measurement strategy is therefore as follows. First a location at some distance $\mathrm{z}$ from the e-beam waist is chosen depending on the beam and available laser parameters. Varying the intensity of the laser allows the optimal laser intensity to be set by optimizing the modulation depth,
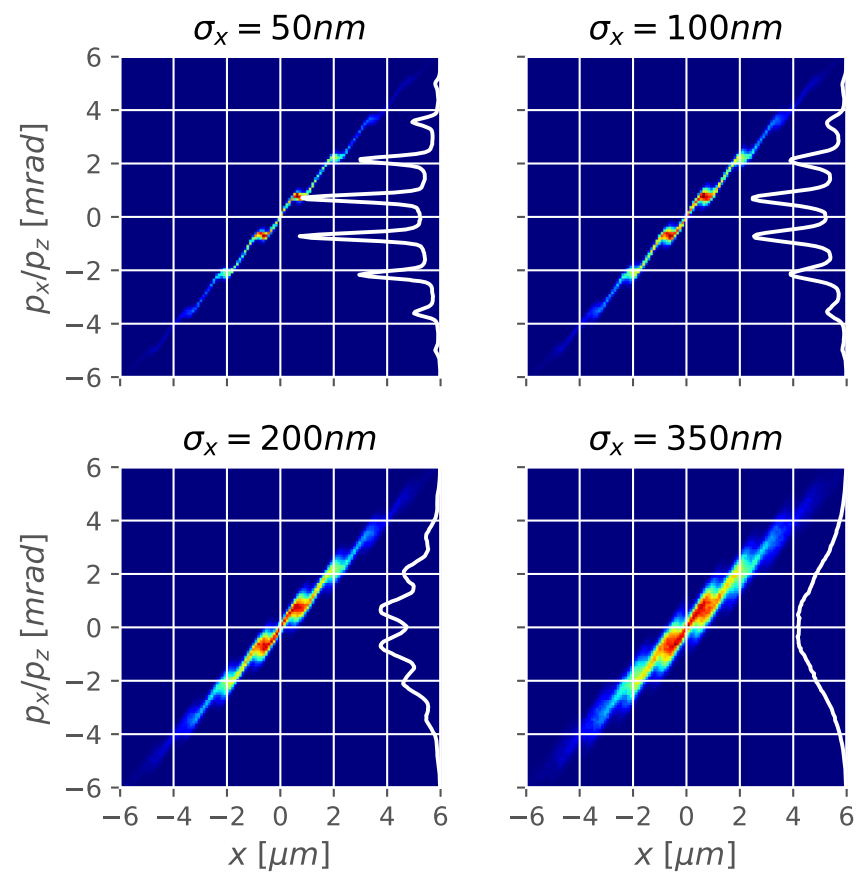

FIG. 6. Momentum space of electron beam $\left(\gamma_{e}=100\right.$, $\left.\sigma_{x^{\prime}}=2 \mathrm{mrad}, \sigma_{z}=2 \mu \mathrm{m}\right)$ after interaction with laser $\left(I_{0}=\right.$ $3.5 \times 10^{16} \mathrm{~W} / \mathrm{cm}^{2}$ ) for different electron source sizes $\sigma_{x}$. White curve: transverse momentum space integrated along $\mathrm{x}$ (measured signal on detector). 
thereby eliminating any systematic effects such as small offsets in achieved actual laser intensity from nominal or distance $\mathrm{z}$ from the electron beam waist. It should be noted here that deviations from the optimal laser intensity of $<10 \%$ did not lead to measurable changes in the simulations peak height (see Fig. 4). At the optimum intensity and for a known interference period $d$ the modulation depth measured in a single shot allows the beam waist size to be directly inferred from the modulated beam profile by comparing with the PIC-simulation/analytical solution which leads to the normalized emittance with $\epsilon_{n}=\beta_{e} \gamma_{e} \sigma_{p x 0} \sigma_{x 0}$. Our analysis has shown this comparison also applies for a laser profile with minor deviations to the theoretically assumed Gauss profile. We compared versions of the analytical solution in which noise of up to $20 \%$ was added to the ponderomotive force to the version without
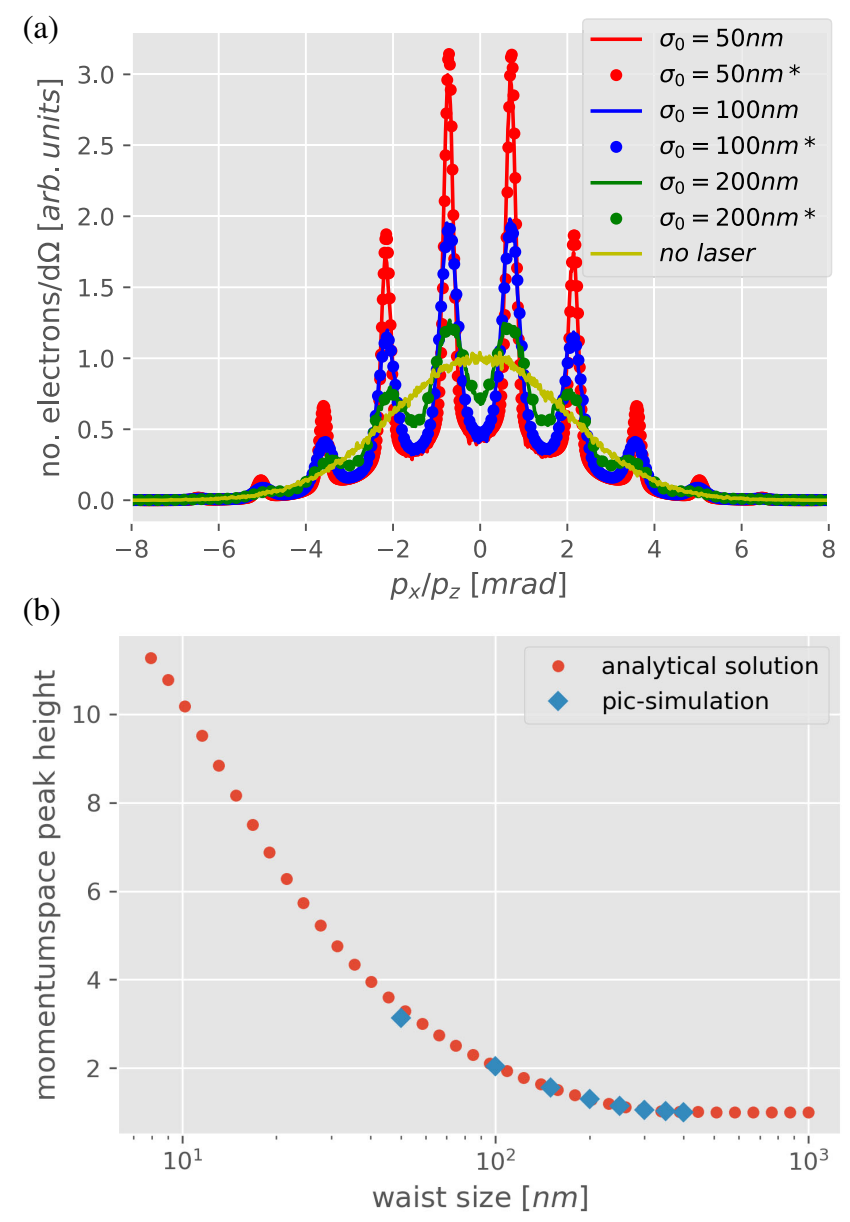

FIG. 7. (a) Transverse momentum space of electron beams with different $\sigma_{x}\left(\sigma_{x^{\prime}}=2 \mathrm{mrad}, \sigma_{z}=2 \mu \mathrm{m}, \gamma_{e}=100\right.$ and $d=0.8 \mu \mathrm{m})$ after interaction. The solid line indicates the PIC simulation results and the dashed the solution of Eq. (5) assuming a sinusoidal electrical laser field. (b) Normalized peak height of modulation in transverse momentum space after laser-electron interaction calculated with Eq. (5). No interaction results in a height of 1 . and observed no measurable difference in modulation depth $\left(\sigma_{x}>0.05 \cdot d\right)$.

Figure 7 compares the calculation of the modulation from the analytical considerations above to the PIC simulations. Clearly the source size can be sensitively determined over a large range with a single measurement configuration. The measurement sensitivity can be further increased by reducing the interference period $d$. Note that for the smallest waist size $(50 \mathrm{~nm})$ in the simulation, which corresponds to an emittance of $10 \mu \mathrm{m} \mathrm{mrad,} \mathrm{an} \mathrm{electron}$ detection system with a resolution better than $40 \mu \mathrm{rad}$ would be required.

This method is capable of characterizing very small waist size beams with unprecedented emittance accurately. The sensitivity of the emittance measurement increases for lower divergence electron beams. Electron beam waists down to tens of nm can be resolved using this technique corresponding to normalized emittance of the order of $\mu \mathrm{m}$ mrad. Using current laser technology it is possible to characterize $\mathrm{GeV}$ e-beams, thereby providing a precise technique to characterize ultralow emittance electron beams under development. In principle this technique can also accommodate beams with significant energy spread, such as those from wakefield accelerators by combining the beam modulation in one plane with a dipole magnet dispersing the electron beam in the other plane. Simulations for a polychromatic electron bunch with an energy spread of $<5 \%$ resulted in no measurable changes in the peak height, which agrees with the sensitivity of the modulation depth to deviations from the optimal intensity. We note that while this technique becomes more demanding in terms of laser intensity at higher electron beam $\gamma_{e}$ it can still be applied and required laser beam energy can be reduced by using sufficiently small spots combined with scanning measurements across the beam spatial dimensions.

\section{CONCLUSION}

We have described a novel scheme for characterizing the properties of an electron beam. To our knowledge, the method is unique in allowing the measurement of extremely small broadband electron beam source sizes and emittances in the $\mu \mathrm{m}$ mrad regime predicted to be accessible using advanced accelerator techniques. Our simulations have shown that emittances as small as $\epsilon_{n}=0.01 \mathrm{~mm}$ mrad can be well resolved. However, this value does not represent an obvious lower limit and can be further reduced by adjusting the parameters appropriately. We note that the method is suited for monoenergetic bunches (from rf linacs) and LWFA bunches with broader bandwidth. The high temporal resolution of the method has the potential to allow slice emittance to be determined for different parts of the beam along the propagation axis, given a monotonic time-energy correlation in longitudinal phase space and a laser pulse duration which is shorter than the electron bunch length. Cross calibration with wellestablished emittance measurement methods is possible 
using typical Ti:sapphire laser systems and electron beams with a transverse beam waist $\sigma_{x 0}<2 \mu \mathrm{m}$.

\section{ACKNOWLEDGMENTS}

This research was funded by the Federal Ministry of Education and Research of Germany in the Verbundforschungsframework (Project No. 05K16SJB).

[1] J. Buon, in Cas Cern Accelerator School-Basic Course 4 (Cern, Geneva, 1990), pp. 30-52.

[2] W. A. Barletta, J. Bisognano, J. N. Corlett, P. Emma, Z. Huang, K.-J. Kim, R. Lindberg, J. B. Murphy, G. R. Neil, D. C. Nguyen, C. Pellegrini, R. A. Rimmer, F. Sannibale, G. Stupakov, R. P. Walker, and A. A. Zholents, Free electron lasers: Present status and future challenges, Nucl. Instrum. Methods Phys. Res., Sect. A 618, 69 (2010).

[3] F. Ji, J. G. Navarro, P. Musumeci, D. B. Durham, A. M. Minor, and D. Filippetto, Knife-edge based measurement of the 4D transverse phase space of electron beams with picometer-scale emittance, Phys. Rev. Accel. Beams 22, 082801 (2019).

[4] D. Marx, J. G. Navarro, D. Cesar, J. Maxson, B. Marchetti, R. Assmann, and P. Musumeci, Single-shot reconstruction of core 4D phase space of high-brightness electron beams using metal grids, Phys. Rev. Accel. Beams 21, 102802 (2018).

[5] S. Rimjaem, G. Asova, J. Bähr, H. J. Grabosch, L. Hakobyan, M. Hänel, Y. Ivanisenko, M. Khojoyan, G. Klemz, M. Krasilnikov et al., in Proceedings of the 32nd Free Electron Laser Conference, Malmö, Sweden (Max-lab, Sweden, 2010).

[6] E. Esarey, C. B. Schroeder, and W. P. Leemans, Physics of laser-driven plasma-based electron accelerators, Rev. Mod. Phys. 81, 1229 (2009).

[7] M. C. Downer, R. Zgadzaj, A. Debus, U. Schramm, and M. C. Kaluza, Diagnostics for plasma-based electron accelerators, Rev. Mod. Phys. 90, 035002 (2018).

[8] A. Pak, K. A. Marsh, S. F. Martins, W. Lu, W. B. Mori, and C. Joshi, Injection and Trapping of Tunnel-Ionized Electrons into Laser-Produced Wakes, Phys. Rev. Lett. 104, 025003 (2010).

[9] C. McGuffey, A. G. R. Thomas, W. Schumaker, T. Matsuoka, V. Chvykov, F. J. Dollar, G. Kalintchenko, V. Yanovsky, A. Maksimchuk, K. Krushelnick, V. Y. Bychenkov, I. V. Glazyrin, and A. V. Karpeev, Ionization
Induced Trapping in a Laser Wakefield Accelerator, Phys. Rev. Lett. 104, 025004 (2010).

[10] T.-Y. Chien, C.-L. Chang, C.-H. Lee, J.-Y. Lin, J. Wang, and S.-Y. Chen, Spatially Localized Self-Injection of Electrons in a Self-Modulated Laser-Wakefield Accelerator by Using a Laser-Induced Transient Density Ramp, Phys. Rev. Lett. 94, 115003 (2005).

[11] J. Faure, C. Rechatin, A. Norlin, A. Lifschitz, Y. Glinec, and V. Malka, Controlled injection and acceleration of electrons in plasma wakefields by colliding laser pulses, Nature (London) 444, 737 (2006).

[12] R. Weingartner, S. Raith, A. Popp, S. Chou, J. Wenz, K. Khrennikov, M. Heigoldt, A. R. Maier, N. Kajumba, M. Fuchs, B. Zeitler, F. Krausz, S. Karsch, and F. Grüner, Ultralow emittance electron beams from a laser-wakefield accelerator, Phys. Rev. ST Accel. Beams 15, 111302 (2012).

[13] G. R. Plateau, C. G. R. Geddes, D. B. Thorn, M. Chen, C. Benedetti, E. Esarey, A. J. Gonsalves, N. H. Matlis, K. Nakamura, C. B. Schroeder, S. Shiraishi, T. Sokollik, J. van Tilborg, C. Toth, S. Trotsenko, T. S. Kim, M. Battaglia, T. Stöhlker, and W. P. Leemans, Low-Emittance Electron Bunches from a Laser-Plasma Accelerator Measured using Single-Shot X-Ray Spectroscopy, Phys. Rev. Lett. 109, 064802 (2012).

[14] B. Hidding, G. Pretzler, J. B. Rosenzweig, T. Königstein, D. Schiller, and D. L. Bruhwiler, Ultracold Electron Bunch Generation via Plasma Photocathode Emission and Acceleration in a Beam-Driven Plasma Blowout, Phys. Rev. Lett. 108, 035001 (2012).

[15] F. Li, J. F. Hua, X. L. Xu, C. J. Zhang, L. X. Yan, Y. C. Du, W. H. Huang, H. B. Chen, C. X. Tang, W. Lu, C. Joshi, W. B. Mori, and Y. Q. Gu, Generating High-Brightness Electron Beams via Ionization Injection by Transverse Colliding Lasers in a Plasma-Wakefield Accelerator, Phys. Rev. Lett. 111, 015003 (2013).

[16] J. Yan, M. Oroku, Y. Yamaguchi, T. Yamanaka, Y. Kamiya, T. Suehara, S. Komamiya, T. Okugi, N. Terunuma, T. Tauchi, S. Araki, and J. Urakawa, Shintake monitor nanometer beam size measurement and beam tuning, Phys. Procedia 37, 1989 (2012).

[17] K. Floettmann, Some basic features of the beam emittance, Phys. Rev. ST Accel. Beams 6, 034202 (2003).

[18] T. D. Arber, K. Bennett, C.S. Brady, A. LawrenceDouglas, M. G. Ramsay, N. J. Sircombe, P. Gillies, R. G. Evans, H. Schmitz, A. R. Bell, and C. P. Ridgers, Contemporary particle-in-cell approach to laser-plasma modelling, Plasma Physics Controlled Fusion 57, 113001 (2015). 